ISSN: 2320-7167

Volume 10 Issue 03 March 2022, Page no. - 2603-2608

Index Copernicus ICV: 57.55, Impact Factor: 7.184

\title{
A Hybrid Fuzzy Logic and Deep Learning Method for Fraud Detection: Application to Credit Cards
}

\author{
Alaa Khouloud ${ }^{1}$, Atounti Mohamed ${ }^{2}$, Bailoul Charaf Eddine ${ }^{3}$ \\ ${ }^{1,2}$ Applied Mathematics and Information Systems Laboratory, Mohammed First University, Nador, Morocco \\ ${ }^{3}$ Applied Mathematics and Computer Science Laboratory, Cadi Ayyad University, Marrakech, Morocco
}

\begin{tabular}{ll}
\hline ARTICLE INFO & ABSTRACT \\
\hline Published Online: & The credit card has become an essential part of business operations, both nationally and \\
02 March 2022 & globally. It plays a very important role in today's economy. While the use of credit cards offers \\
& considerable benefits when used responsibly and with care, considerable financial and credit \\
& damage can be caused by fraudulent default. Many techniques have been proposed to combat \\
& the growth of credit card defaults. However, all these techniques have the same goal of \\
& preventing credit card defaults; each has its own characteristics, advantages, and \\
& disadvantages. In this paper, we applied two data mining techniques: Artificial Neural \\
& Networks and Fuzzy C-Means to this problem, we propose a hybridization between these two \\
& models and also, we show significant results of the three methods on real financial data. \\
& Therefore, out of all the methods used, good performances are obtained by using our hybrid \\
model to fraud detection.
\end{tabular}

KEYWORDS: Fraud detection; Classification; Data mining; Deep learning; Fuzzy c-means; Confusion matrix

\section{INTRODUCTION}

An important business research question is to classify companies into different pre-defined groups [9], which can be used as an effective risk management tool. Prediction of payment default fraud has been a major field of commercial interest for many researchers, both practically and theoretically [9], because it is an integral part of credit risk, which is considered as one of the most important banking risks [13]. Expert prediction of payment default fraud is of interest to a large range of stakeholders such as banks, microcredit organizations, insurance companies, other creditors, auditors, etc. The increase in default fraud cases can be linked to poor credit risk management and the latest global financial crisis.

The global convergence of capital standards and measures separates the minimum capital requirements for banks into three main parts. The three main elements used to define minimum capital requirements are credit risk, operational risk and market risk.

The main interest of this research is the evaluation of the probability of occurrence of payment default. Payment default, as defined by the Basel Committee on Banking
Supervision, occurs when one or more of the following events occur:

- The debtor is determined to be unlikely to pay his or her debts in full (principal, interest or fees).

- An event of credit loss associated with a debtor's obligation, such as a charge, a specific provision or a difficult restructuring involving the forgiveness or deferral of principal, interest or fees.

- The debtor is more than 90 days past due on any credit obligation.

- The debtor has filed for bankruptcy or similar protection against creditors.

Financial institutions are augmenting the financial facilities that are currently available through innovative services such as credit cards, mobile banking, ATMs and the Internet. In addition, with the rapid advancement of e-commerce, the use of credit cards has become a necessary and convenient part of financial life. A credit card is a payment card that is provided to customers as a payment system.

In order to predict credit default payment fraud, several classification techniques are used in the existing literature. 
The aim of this study is to hybridize two data mining methods: Artificial Neural Networks (ANNs) and Fuzzy CMeans (FCMs), to increase the performance and reduce the misclassification rate. After a learning process, the program is supposed to be able to correctly classify a customer who has not been previously seen as risky or not risky, considering certain attributes of this customer.

This article is structured as follows: first, we introduce the readers to the area of fraud detection. In section 2, we explain the two techniques used in this paper (ANNs and FCM). Lastly, we present our new hybrid algorithm and discuss the experiments performed for these models, followed by a conclusion.

\section{LITERATURE REVIEW}

In the information explosion era, companies are producing and collecting huge volumes of data on a daily basis. The main challenge for companies is to convert this information into usable results and discover useful information from the database. The process of exploration and analysis of huge volumes of data, to discover significant patterns and rules, is data mining. It is an essential tool in the decision-making system, playing a key role in default detection, credit assessment, market segmentation, customer services and benchmarking [12,15]. The pros and cons of the two techniques of data mining used in this paper are discussed $[6,11,12]$.

\section{A. Artificial Neural Networks}

ANNs are modeled by the human brain and consist of a large number of artificial neurons, but the neurons in ANNs have fewer roles and connections and are less numerous than biological neurons; however, the functioning of both networks is the same. First, as in an ANN network, inputs are received from other connected neurons or perceptron at the other end of the network. An activation function is then assigned to these received inputs, which leads to the activation level of the neuron, which is actually the neuron's output value. There are different activation functions which can be applied to generate non-linearity in the neural network, like sigmoid function, step function, linear function.

A deep neural network (DNN) is made up of more than three layers of neurons, input layer, hidden layers and output layer. It is based on the idea of consecutive layers, these layers determining the depth of the model. DNNs are a form of multilayer feedforward network (MLP) in which data flows from the input layer to the output layer.

Figure 1 is a multilayer perceptron model which consists of four layers: first layer is the input layer, two hidden layers and the last layer is the output layer.

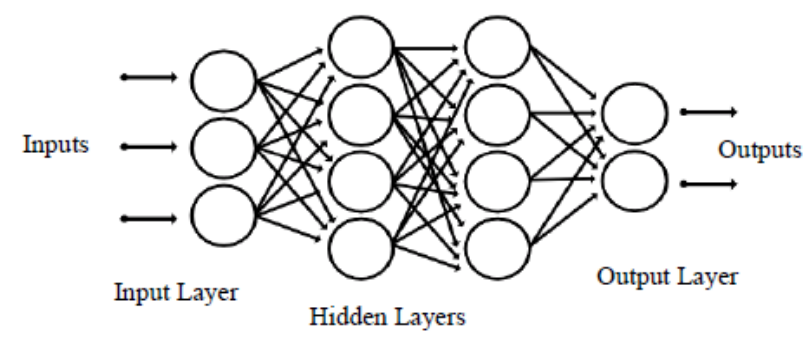

Figure 1: A multilayer perceptron network.

In order to develop significant relationships between inputs and outputs via a learning process, DNNs use nonlinear mathematical equations [1]. Backpropagation first appeared in 1970, but it is considered the workhorse of neural network learning in the present environment. A core algorithm in a DNN to develop a meaningful relationship between inputs and outputs through the learning process.

Backpropagation is an algorithm used to find the minimum value of the error function by adjusting weights using the delta rule or gradient descent. Figure2 shows how backpropagation works.

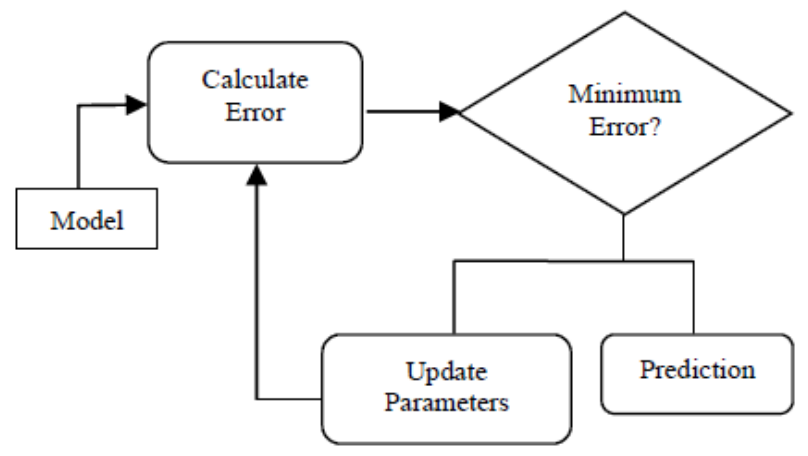

Figure 2: Backpropagation.

DNNs can easily handle both interactive and non-linear impacts. The main disadvantage of DNNs is that they cannot provide a simple probabilistic classification formula.

\section{B. Fuzzy C-Means}

Fuzzy clustering is a powerful unsupervised method for modeling and data analysis. Fuzzy clustering is much more natural than hard clustering in most cases. Objects located at the boundary of several classes are not obliged to fully belong to one of them but are attributed membership degrees between 0 and 1 to indicate their partial membership. The FCM algorithm is the most commonly used.

FCM clustering was first introduced in the literature for a particular case $(m=2)$ by Joe Dunn in 1974. The general case (for any $\mathrm{m}$ greater than 1) was developed by Jim Bezdek in his PhD thesis at Cornell University in 1973. It was improved by Bezdek in 1981. FCM utilizes fuzzy clustering so that a data point can be classified into all groups with different membership degrees between 0 and 1 $[5,7]$. 


\section{Algorithm 1:}

1. Initialize $U=[u(i, j)]$ matrix, $U(0)$

2. At k-step : calculate the centers vectors $C(k)=[c(j)]$ with $\mathrm{U}(\mathrm{k})$

$$
C_{i}=\frac{\sum_{j=1}^{n} u_{i j}{ }^{m} x_{j}}{\sum_{j=1}^{n} u_{i j}{ }^{m}}
$$

3. Update $\mathrm{U}(\mathrm{k}), \mathrm{U}(\mathrm{k}+1)$

4.

$$
\begin{gathered}
d_{i j}=\sqrt{\sum_{i=1}^{n}\left(x_{i}-c_{i}\right)} \\
u_{i j}=\frac{1}{\sum_{k=1}^{c}\left(\frac{d_{i j}}{d_{k j}}\right)^{2 /(m-1)}}
\end{gathered}
$$

5. If $\|\mathrm{U}(\mathrm{k}+1)-\mathrm{U}(\mathrm{k})\|<\varepsilon$, then STOP ; otherwise return to step 2 .

Here $m:$ is any real number greater than 1 , $\mathrm{u}(\mathrm{i}, \mathrm{j})$ : is the degree of membership of $\mathrm{x}(\mathrm{i})$ in the cluster $j$, $\mathrm{x}(\mathrm{i})$ : is the $i$ th of d-dimensional measured data,

$c(j)$ : is the d-dimension center of the cluster.

\section{Proposed Algorithm}

The hybrid approach towards default payment fraud is best modelled by the prototype as shown in Figure 3.

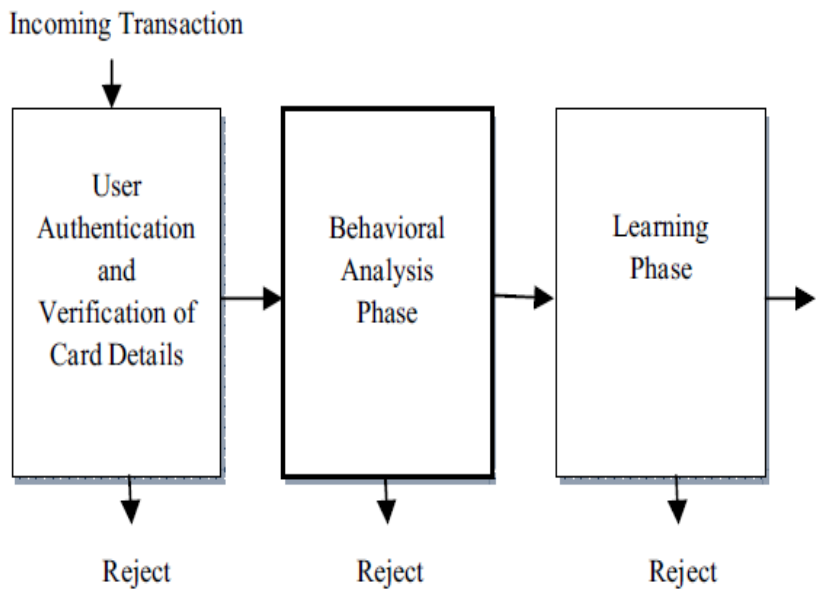

Figure 3: Prototype structure

Our new hybrid algorithm consists of the following three phases:

- Initial Authentication and Verification :

The first layer is the layer for initial verification, screening and where user authenticity is verified.

- Behavioural Analysis Phase :

This phase consists in applying the FCM method to our data in order to obtain an output $\mathrm{U}$. The $\mathrm{u}(\mathrm{i}, \mathrm{j})$ is then compared to the already preset threshold values, namely the upper threshold $(\mathrm{u}(\mathrm{Uth}))$ and the lower threshold $(\mathrm{u}(\mathrm{Lth}))$ which are experimentally determined. Depending on the result of the comparison, three rules are defined as follows:
- If $(u(i, j)<u(L t h))$, then the customer is nonrisky.

- Else if $(\mathrm{u}(\mathrm{Lth})<\mathrm{u}(\mathrm{i}, \mathrm{j})<\mathrm{u}(\mathrm{Uth}))$, then move the customer to the suspicious table for applying the learning mechanism for strengthening the initial observation.

○ Else, reject the customer i.e. when $u(i, j)>u($ Uth $)$.

The customers those are found to be suspicious are passed to the learning phase before taking the final decision.

- Behavioural Analysis Phase :

Suspicious customers are then moved to the suspect table where they are kept for further analysis and classification that is performed using feedforward neural networks with backpropagation.

\section{CLASSIFICATION ACCURACY}

\section{A. Description of the Data}

This study took payment data from a big bank in October 2005 (a credit card and cash issuer) in Taiwan. Out of a total of 30000 observations, 5529 customers are risky. This research uses a binary variable - default payment (Yes $=1$, No $=0$ ), as the response variable. Our study uses 23 variables as explanatory variables:

- X1: Amount of the credit (NT dollar). It includes both individual consumer credit and family credit.

- X2: Gender $(1=$ male $; 2$ = female $)$.

- $\mathrm{X} 3$ : Education $(1=$ graduate school; 2 = university $; 3=$ high school ; 4 = others).

- $\mathrm{X} 4$ : Marital status ( $1=$ married; $2=$ single $; 3=$ others $)$.

- X5 : Age (year).

- X6-X11: History of past payment. We tracked the previous monthly payment records (from April to September, 2005) as follows: X6 = the repayment status in September, 2005; X7 = the repayment status in August, 2005; . . . ; X11 = the repayment status in April, 2005.

The reimbursement status measurement scale is: $-1=$ pay duly ; 1 = payment delay for one month ; $2=$ payment delay for two months $; \ldots ; 8$ = payment delay for eight months ; 9 = payment delay for nine months and above.

- X12-X17: Invoice amount (NT dollar). X12 = amount of bill statement in September, 2005; X13 = amount of bill statement in August, 2005; . . . ; X17 = amount of bill statement in April, 2005.

- X18-X23: Previous payment amount (NT dollar). X18 = amount paid in September, 2005; X19 = amount paid in August, 2005; . . . ; X23 = amount paid in April, 2005.

We are especially interested in knowing how many risky clients are correctly classified as risky and, simultaneously, how many non-risky clients are incorrectly classified as risky. The former is called the true positive rate and the latter the false positive rate. 


\section{B. Results}

This section shows the simulation results of the two mentioned models as well as of the proposed hybrid method. To better visualize the results obtained by the best model (DNNs), we used the confusion matrix which is a very famous measure used for solving classification problems. It can be applied to binary classification as well as to multiclasses classification problem (see Table 1).

Table 1: Confusion matrix

\begin{tabular}{|c|c|c|c|c|c|}
\hline Confusion & & Predic & class & & \\
\hline $\begin{array}{l}\text { Actual } \\
\text { class }\end{array}$ & 0 & $\begin{array}{l}\text { True } \\
\text { (TP) }\end{array}$ & positive & $\begin{array}{l}\text { False } \\
(\mathrm{FN})\end{array}$ & negative \\
\hline & 1 & $\begin{array}{l}\text { False } \\
\text { (FP) }\end{array}$ & positive & $\begin{array}{l}\text { True } \\
\text { (TN) }\end{array}$ & negative \\
\hline
\end{tabular}

The accuracy, sensitivity, and specificity are also used to evaluate models' performance, as follows:

Accuracy $=(\mathrm{TP}+\mathrm{TN}) /(\mathrm{TP}+\mathrm{FN}+\mathrm{FP}+\mathrm{TN})$

Sensitivity $=\mathrm{TP} /(\mathrm{TP}+\mathrm{FN})$

Specificity $=\mathrm{TN} /(\mathrm{FP}+\mathrm{TN})$

The deep network architecture used here is given in Figure5: we have used a deep network with three hidden layers, the first contains 10 neurons, the second 15 neurons and the last 10 neurons.

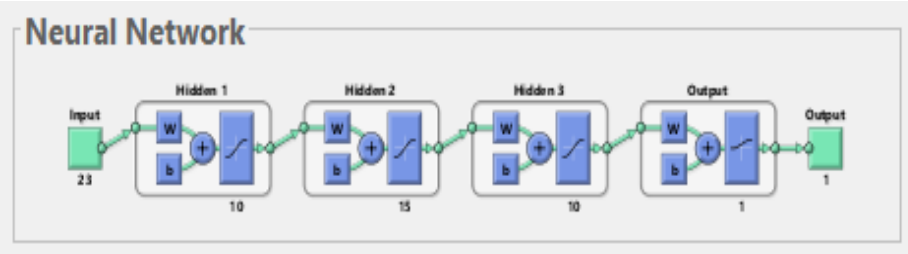

Figure 4: Deep network architecture

In the confusion matrix graph, the columns represent the actual class (target class) and the rows represent the predicted class (output class). The off-diagonal cells correspond to cases that are misclassified. The diagonal cells correspond to cases that are correctly classified. The number of observations and the percentage of the total number of observations are given in each cell.

The bottom line of the graph indicates the percentages of all observations in each class which are correctly and incorrectly classified. These measures are often called recall (or true positive rate) and false negative rate, respectively. The column on the extreme right of the graph gives the percentages of all observations that are predicted to belong to each class that are correctly and incorrectly classified. These measurements are often referred to as accuracy (or positive predictive value) and false detection rate, respectively. The bottom right cell of the graph shows the overall accuracy.
By applying FCM algorithm to our data, we obtain the confusion matrix shown in Figure 6. As we see, $76.6 \%$ of the predictions are correct and $23.4 \%$ are wrong.

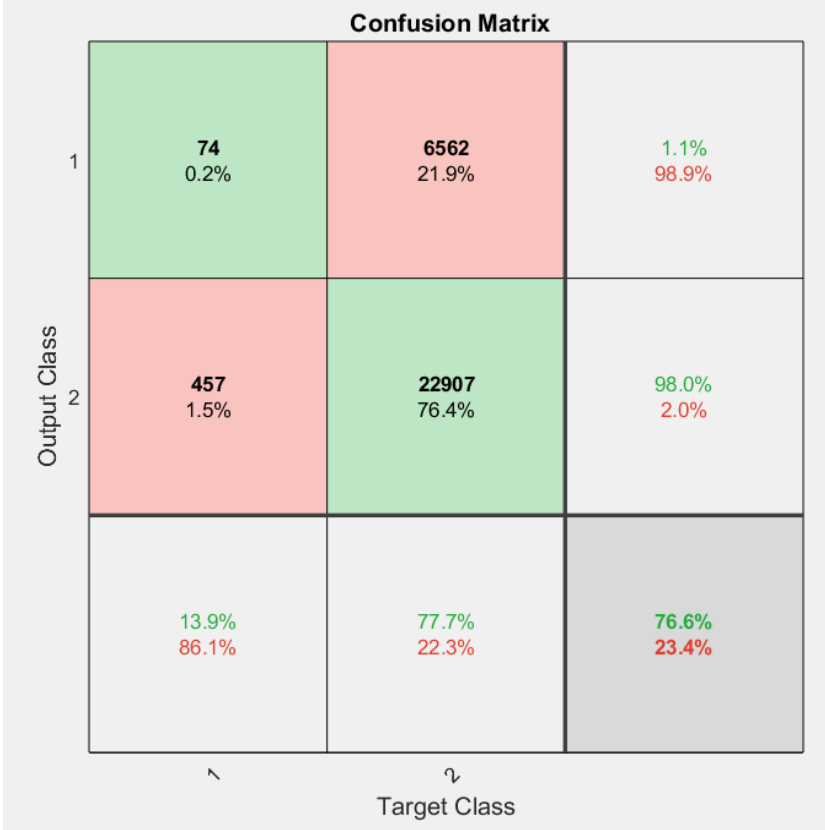

Figure 5: The confusion matrix obtained by FCM model

With the ANNs method, we obtain the confusion matrix presented in Figure 7, we get a performance of $78 \%$.

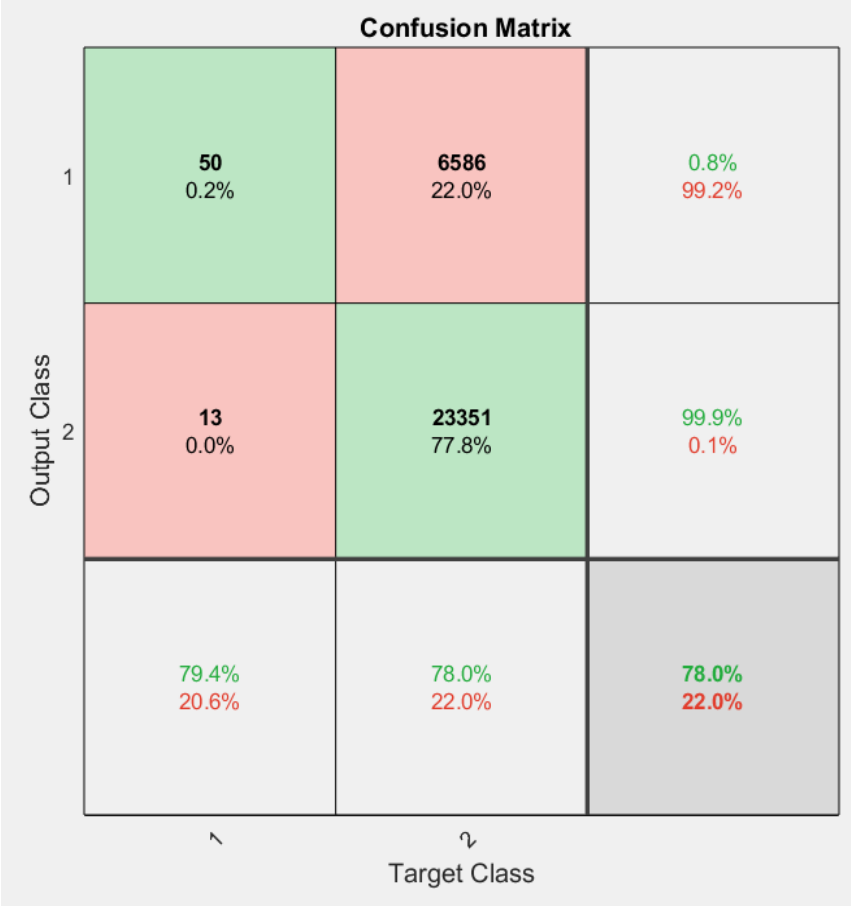

Figure 6: The confusion matrix obtained by ANNs model

Using our proposed hybrid algorithm, we get very satisfactory results (see Figure 8). 


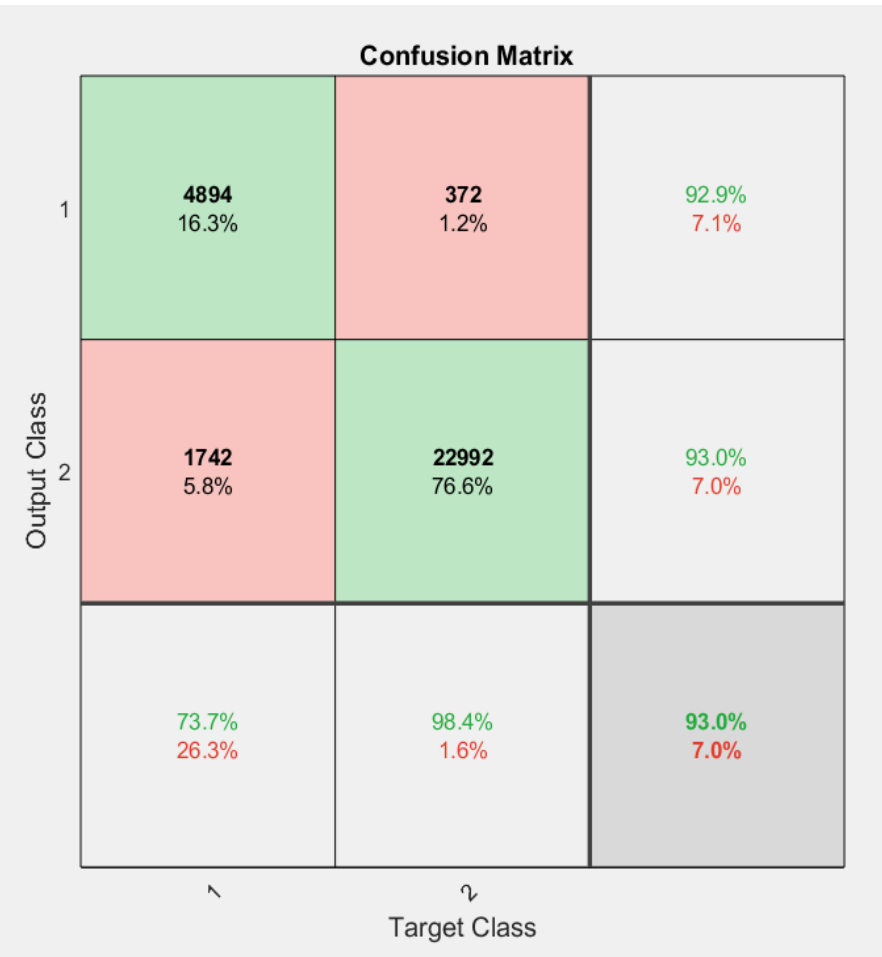

Figure 7: The confusion matrix obtained by the proposed model

The first and second diagonal cells represent the number and percentage of proper classifications achieved by the trained network.

For example, 22992 customers are correctly classified as non-risky, this corresponds to $76.6 \%$ of all the data. Similarly, 4894 customers are correctly classified as risky and this corresponds to $16.3 \%$ of our data. 1742 customers are incorrectly classified as risky and this corresponds to $5.8 \%$ of the data. Similarly, 372 customers are incorrectly classified as non-risky and this corresponds to $1.2 \%$ of our data.

Out of 24734 non-risky customers, 93\% are correct and 7\% are wrong. Out of 5266 risky customers, $92.9 \%$ are correct and $7.1 \%$ are wrong. Out of 23364 non-risky cases, $98.4 \%$ are correctly predicted as non-risky and $1.6 \%$ are predicted as risky. And out of 6636 risky cases, $73.7 \%$ are correctly classified as risky and $26.3 \%$ are classified as non-risky. Overall, $93 \%$ of the predictions are correct and $7 \%$ are wrong. It is to note that the hybrid algorithm is more accurate and efficient in comparison with the two other techniques.

Table 2: Classification results

\begin{tabular}{|l|l|l|l|}
\hline Model & Accuracy & Sensitivity & Specificity \\
\hline FCM & 0.766 & 0.011 & 0.980 \\
\hline ANNs & 0.780 & 0.007 & 0.999 \\
\hline Hybrid & 0.930 & 0.929 & 0.930 \\
\hline
\end{tabular}

The values of the parameters used in the performance evaluation of the three models are presented in Table 2.
The best results are obtained by our proposed hybrid algorithm.

\section{CONCLUSION}

While default payment fraud prevention has received much attention in the literature, researchers still face problems that have not been addressed in the proper manner. This article reviews two main classification techniques: Artificial Neural Networks and Fuzzy C-Means, it proposes a hybridization of these two models and compares the classification performance and predictive accuracy between them. This research work performed data preprocessing, normalization, and subsampling to address the problems faced when using an imbalanced data set. The results show that our hybrid algorithm performs more accurate classification than other models on credit card fraud.

\section{REFERENCES}

1. Asha, R.B., and Suresh Kumar, K.R., 2021, "Credit Card Fraud Detection using Artificial Neural Network", Global Transitions Proceedings, january, 2, 5-41.

2. Basel Committee on Banking Supervision\}. International Convergence of Capital Measurement and Capital Standards: A revised framework comprehensive version, 2006, Bank of International Settlements.

3. Bezdek, J.C., 1981, "Pattern Recognition with Fuzzy Objective Function Algorithms”, Plenum Press, New York.

4. Dunn, J.C., 1973, "A Fuzzy Relative of the ISODATA Process and Its Use in Detecting Compact Well-Separated Clusters", EJournal of Cybernetics, 3, 32-57.

5. Eyoh, I., Eyoh, J., Umoh, U. and Kalawsky, R., 2021, "Optimization of Interval Type-2 Intuitionistic Fuzzy Logic System for Prediction Problems", International Journal of Computational Intelligence and Applications. 20(4).

6. Han, J., Kamber, M., 2001, "Data Mining: Concepts and Techniques", San Francisco: Morgan Kaufmann.

7. Hassanzadeh, T., Meybodi, M.R. and Shahramirad, M., 2017, "A New Fuzzy Firefly Algorithm with Adaptive Parameters", International Journal of Computational Intelligence and Applications, 16(3).

8. Hunter, C.W., Kaufman, G.G. and Krueger, T.H., 1991, "The Asian Financial Crisis: Origins, Implications, and Solutions", Springer.

9. Jo, H. and Han, I., 1996, "Integration of Case Based Forecasting, Neural Network, and Discriminant Analysis for Bankruptcy Prediction”, Expert Systems with Applications, 11(4), 415-422. 
"A Hybrid Fuzzy Logic and Deep Learning Method for Fraud Detection: Application to Credit Cards"

10. Muller, G.H., Steyn-Bruwer, B.W., and Hamman, W.D., 2009, "Predicting Financial Distress of Companies Listed on The JSE - Comparison of Techniques", South African Journal of Business Management, 40(1), 21-32.

11. Padma, S. and Pugazendi, R., 2018, "Solving Classification Problems Using Projection-Based Learning Algorithm with Fuzzy Radial Basis Function Neural Network", International Journal of Computational Intelligence and Applications, 17(3).

12. Paolo, G., 2003, "Applied Data Mining: Statistical Methods for Business and Industry", New York: John Wiley \& Sons, Inc.

13. Sarlija, N., Pejic-Bach, M. and Jakovic, B., 2008, "Managing Credit Risk in Croatian Banking Sector. In Wagner, U. et al., eds.: 28th Scientific Symposium "The Future of Monetary and Financial Stability in Europe", Fakultat fur Wirtschaft und Recht \& Faculty of Economics, Osijek, 125-140.

14. Tengke, X, Shengrui, W., Mayers, A. and Monga, E., 2013, "Personal Bankruptcy Prediction by Mining Credit Card Data", Expert Systems with Applications, 40(2), 665-676.

15. Thomas, L.C., 2000, "A Survey of Credit and Behavioral Scoring: Forecasting Financial Risk of Lending to Consumers", International Journal of Forecasting, 16, 149-172. 